\title{
Monopolar Spin Orientation and Determination of Spin Relaxation Times in Quantum Well Structures.
}

Sergey D. Ganichev ${ }^{1,2}$, Sergey N. Danilov ${ }^{1}$, Martin Sollinger ${ }^{1}$, Dieter Weiss ${ }^{1}$, Werner Wegscheider ${ }^{1}$, Wilhelm Prettl ${ }^{1}$, Vasily V. Bel'kov ${ }^{2}$, and Eugenius L. Ivchenko ${ }^{2}$,

${ }^{1}$ Fakultät für Physik, Universität Regensburg, 93040 Regensburg, Germany,

${ }^{2}$ A. F. Ioffe Physicotechnical Institute, 194021 St. Petersburg, Russia

\begin{abstract}
It is shown that monopolar optical spin orientation of free carriers in zinc-blende structure based quantum wells (QWs) causes an electric current which reverses its direction upon changing the helicity of the radiation from left to right circular polarization resulting in a circular photogalvanic effect. The monopolar non-equilibrium population of spin-up and spin-down states has been achieved by far-infrared optical excitation of $p$ - and $n$-type GaAs/AlGaAs QWs structures. Two methods are introduced allowing to determine spin relaxation times. One is based on the Hanle effect in magnetic field induced circular photogalvanic effect, the other is spin sensitive bleaching of absorption. In contrast to usually applied methods of optical spin orientation, in the present case of terahertz excitation only one kind of charge carriers is involved in spin orientation and relaxation processes.
\end{abstract}

\section{INTRODUCTION}

A substantial portion of current research in condensed matter physics is directed towards understanding various manifestations of spin dependent phenomena. In particular, the spin of electrons and holes in solid state systems is the decisive ingredient for active spintronic devices (for review see [1]). For the realization of spintronic devices sufficiently long spin dephasing times in semiconductor QW structures are crucially needed. Current investigations of the spin lifetime in semiconductor devices are based on optical spin orientation by interband excitation and further tracing the kinetics of polarized photoluminescence [1-4]. These studies gave important insights into the mechanisms of spin relaxation of photoexcited free carriers.

In low dimensional systems optical excitation by circularly polarized light not only leads to a spin polarized ensemble of electrons but also to a current whose direction depends on the helicity of the incident light [5]. The coupling of the helicity of the incoming photons to spin polarized final states with net momentum is caused by angular momentum selection rules together with band splitting in $\mathrm{k}$-space due to $\mathrm{k}$-linear terms in the Hamiltonian. We show below that investigation of this circular photogalvanic effect (CPGE) in dependence on magnetic field strength or the radiation intensity allows to probe spin relaxation for monopolar spin orientation in $n$ - and $p$-type materials. In contrast to the conventional methods of optical spin orientation, in our measurements only one type of charge carriers gets spin oriented and is involved in relaxation processes. This is achieved by using radiation in the terahertz range which excites intraband or intersubband, but no interband transitions. Thus monopolar spin orientation allows to study spin relaxation without electron-hole interaction and exciton formation in the conditions close to the case of electron spin injection.

\section{SAMPLES AND EXPERIMENTAL TECHNIQUE}

The experiments have been carried out on MBE (001)-grown $n$-GaAs/AlGaAs single heterojunctions and modulated doped $p$-GaAs/AlGaAs (113)-MBE-grown samples with a single QW of the $15 \mathrm{~nm}$ width. Samples with free carrier densities of about $2 \cdot 10^{11} \mathrm{~cm}^{-2}$ were studied in the range from liquid helium to room temperature. A pair of ohmic contacts have been centered on opposite sample edges along the direction $x \|[1 \overline{1} 0]$ (see inset in Fig. 1b). As terahertz radiation source a high power FIR-infrared molecular laser [6], optically pumped by a TEA-CO $\mathrm{CO}_{2}$ laser, has been used delivering $100 \mathrm{~ns}$ pulses with intensities up to $1 \mathrm{MW} / \mathrm{cm}^{2}$ at wavelength $\lambda=148 \mu \mathrm{m}$. The 
(a)

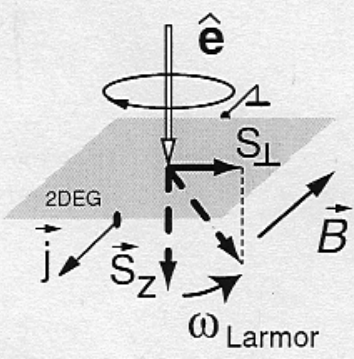

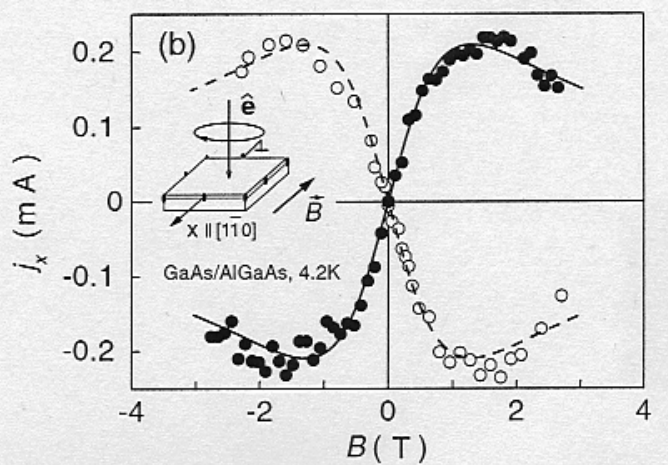

Figure 1. (a) Sketch of optical orientation in the presence of an in-plane magnetic field. The field is perpendicular to the optically aligned spins and rotates the spins by Larmor precession into the plane of the heterojunction. (b) Current $j_{x}$ as a function of magnetic field for normaly incident right-handed (open dots) and left-handed (solid dots) circularly polarized radiation at $\lambda=148 \mu \mathrm{m}$ and $P=20 \mathrm{~kW}$. The inset shows the geometry of the experiment. Curves are fitted after Eq. (1).

radiation induces indirect transitions in the lowest conduction subband of our $n$-type samples and direct transitions between valence subbands in the $p$-type samples. A $\lambda / 4$ plate has been used to obtain from the initially linearly polarized light circularly polarized radiation with the degree of circular polarization $P_{\text {circ }}$ equal to \pm 1 for right and left handed circularly polarized light, respectively.

\section{HANLE EFFECT IN MAGNETIC FIELD INDUCED CPGE}

In this section it is shown that the magnetic field induced CPGE allows to obtain relaxation times of spin oriented carriers via observation of the Hanle effect $[7,8]$. In recent work we have demonstrated that irradiation of quantum wells with circularly polarized light in the presence of external magnetic field in the plane of a heterojunction causes a magnetic field induced CPGE [9]. Microscopically the conversion of spin into the electric current is caused by asymmetric spin-flip relaxation of spin oriented electrons in the system with $k$-linear terms in the effective Hamiltonian. Phenomenologically the electric current $j$ linked to a non-equilibrium spin polarization $S$ can be written as [10] $j_{\alpha}=Q_{\alpha \gamma} S_{\gamma}$ where $Q_{\alpha \gamma}$ is a second rank pseudo-tensor which relates the axial vector $S_{\gamma}$ to the polar vector $j_{\alpha}$. In the (001)-grown low dimensional structures of $C_{2 v}$ symmetry investigated here only two independent components $Q_{x y}$ and $Q_{y x}$ are non-zero. Therefore for the above described effect of an asymmetric spin relaxation induced current $j$ to take place, an in-plane spin component $\boldsymbol{S}_{\perp}$ must exist as depicted in Fig. 1a. We note that this effect does not require optical excitation and can be achieved by any means of spin orientation.

The $n$-type GaAs samples were placed in a magneto-optical cryostat and illuminated by the radiation at normal incidence. As it is shown in [5], for (001)-grown structures as used here, optical orientation yields a spin orientation normal to the film but does not lead to the CPGE without a magnetic field. An in-plane magnetic field $B$ up to $3 \mathrm{~T}$ has been applied as sketched in the inset of Fig. 1b. The magnetic field perpendicular to the optically aligned spins rotates the spins by Larmor precession into the plane of the heterojunction as it is depicted in Fig. 1a. As a result an in-plain component of the spin occurs yielding an in-plain electrical current those direction depends on the orientation of the spin in the plane of interfaces. It reverses it's direction when the circular polarization of the laser beam switches from left-handed to right-handed and also changes sign under magnetic field reversal (Fig. 1b). The current is parallel (antiparallel) to the magnetic field vector. Fig. $1 \mathrm{~b}$ shows the current $j_{x}$ in (001)-grown GaAs/AlGaAs at $4.2 \mathrm{~K}$ as a function of the magnetic field strength $B$ for normal incident right- and left-handed circularly polarized radiation of $P=20 \mathrm{~kW}$. At low magnetic fields up to about $2 \mathrm{~T}$ the current $j_{x}$ rises linearly with the field 
strength $B$, assumes a maximum and then drops with further rising magnetic field. In the absence of a magnetic field and at normal incidence of radiation the current is zero. In the p-type samples no magnetic field induced current has been observed.

In the present experiment where spins were oriented by application of normal incident circularly polarized radiation, the spin generation rate $\dot{\mathbf{S}}$ is described by $\dot{\mathbf{S}} \propto \pm \mathrm{E}^{2} \hat{\mathbf{e}}_{\mathrm{z}}$, where $E$ is the amplitude of the electric field of the light, $\hat{\mathbf{e}}_{\mathbf{z}}$ is the unit vector pointing in the direction of the light propagation and plus and minus sign correspond to right- and left-handed circular polarized radiation, respectively. The in-plane component of the total electron spin $\mathbf{S}_{\perp}$ obtained by Larmor precession is related to the spin generation rate and to the in-plane magnetic field by

$$
\mathbf{S}_{\perp}=\tau_{\mathrm{s}}^{2} \frac{\boldsymbol{\omega}_{L} \times \dot{\mathbf{S}}}{1+\left(\boldsymbol{\omega}_{L} \tau_{\mathrm{s}}\right)^{2}}
$$

where $\tau_{s}=\sqrt{\tau_{s} \| \tau_{s \perp}}$ and $\tau_{s \|}, \tau_{s \perp}$ are the longitudinal and transverse electron spin relaxation times, $\omega_{L}=g \mu_{\mathrm{B}} \mathbf{B} / \hbar$ is the Larmor frequency, $g$ is the electron $g$-factor and $\mu_{B}$ is the Bohr magneton. The decay of $\boldsymbol{S}_{\perp}$ with increasing Larmor frequency above the spin relaxation rate $\tau_{s}^{-1}$ corresponds to the Hanle effect. The Hanle effect, depolarizing spin oriented states, is well known from atomic spectroscopy [7]. It has also been observed in photoluminescence of bulk semiconductors with interband excitation [8] but never in a photocurrent of low dimensional semiconductor structures.

The Hanle effect (see Fig. 1b) gives strong evidence that the current is caused by spin orientation due to free carrier intraband transitions. As another important result, the spin relaxation time being crutial for spintronics may immediately be obtained from the magnetic field strength of the peak current where $\omega_{L} \tau_{s}=1$. The magnitude of the observed spin relaxation time in the first electron subband obtained using the $g$-factor $g=-0.2$ (see [11]) gives $\tau_{s}=40 \mathrm{ps}$ in the range between 2.1 and $4.2 \mathrm{~K}$. This result agrees to previously published data obtained from the interband recombination kinetics of circularly polarized photoluminescence [2-4].

As it is seen from Eq. (1) the spin induced current is proportional to the Larmor frequency. This makes the mechanism inefficient for p-type samples because the in-plane $g$ factor for heavy holes is very small [12]. This explains the fact that no magnetic field induced current has been observed in $p$-type GaAs structures.

\section{SPIN SENSITIVE BLEACHING}

In $p$-type materials where magnetic field induced CPGE is not detected, spin relaxation of holes may be obtained by means of FIR saturation spectroscopy [13-15]. Spin sensitive bleaching of the heavy hole ( $h h 1)$ - light hole (lh1) absorption in p-type QW structures observed here allows to investigate spin relaxation for a monopolar spin orientation. The basic physics is sketched in Figs. 2a and 3a. Exciting with far-infrared light results in direct intersubband transitions (solid arrow in Fig. 2a). The absorption of circularly polarized light is spin selective because only one type of spin component is involved in the absorption process (illustrated in the right bottom inset of Fig. 3a). This process depopulates and populates selectively spin states in the valence subbands $(h h 1$ and $l h 1)$. As the spin relaxation time of photoexcited holes in the $l h 1$ subband is very short [16] spin orientation occurs in the initial subband $h h 1$ only. The absorption coefficient $\alpha$ is proportional to the difference of the populations of the initial and final states. At high intensities the absorption coefficient decreases since the photoexcitation rate becomes comparable to the non-radiative relaxation rate into the initial state. The absorption bleaching of circularly polarized radiation is governed by energy relaxation of photoexcited carriers and hole spin relaxation in the initial state. These processes are characterized by energy and spin relaxation times $\tau_{e}$ and $\tau_{s}$, respectively. In contrast to circularly polarized light, optical transitions induced by linearly polarized light are not spin selective (top left inset in Fig. 3a) and the saturation is controlled by the energy relaxation time of photoexcited carriers $\tau_{e}$ only. Note that $\tau_{e}$ is the same for circular and linear polarization. If $\tau_{s}$ is of the order of $\tau_{e}$ or larger, bleaching of absorption becomes spin sensitive and the saturation intensity of circularly polarized radiation drops below the value of linear polarization. The difference in absorption bleaching for circularly and linearly polarized radiation can be observed experimentally 
(a)

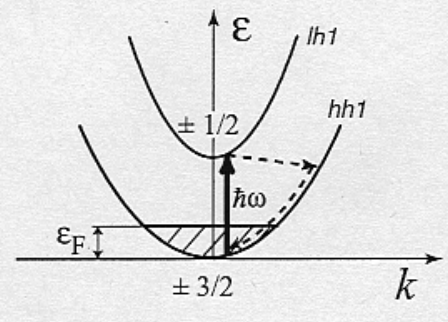

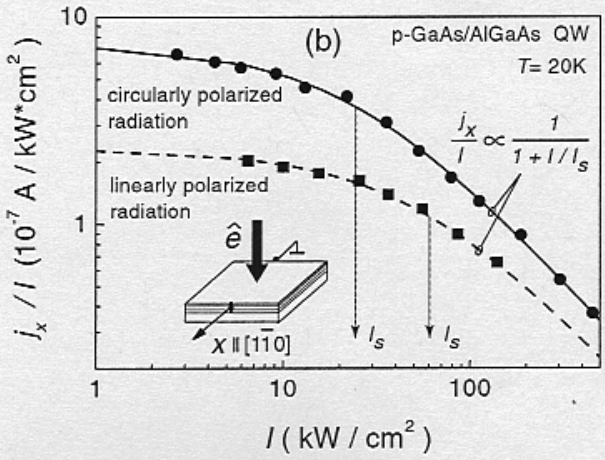

Figure 2. (a) Sketch of direct optical transitions between the first heavy hole and the first light hole subband in p-GaAs/AlGaAs QWs. While the splitting of the bands in k-space is necessary for an understanding of the circular photogalvanic effect [5] it is unimportant for the saturation process and ignored in the sketch. Broken arrows show the energy relaxation of photoexcited carriers. $\varepsilon_{F}$ is the Fermi energy. (b) Photogalvanic current $j_{x}$ normalized by the intensity $I$ as a function of $I$ for circularly and linearly polarized radiation. The inset shows the geometry of the experiment.

as is pointed out below. The magnitude of the saturation intensity allows to derive relaxation times responsible for the bleaching.

The weak ahsorntion nf terahertz radiation hy free sarriers in $\mathrm{OW}$ s is difficult to determine bx 
